\title{
POETRY
}

\section{Steeplechase charity gala}

With a terrific clang, the gates open And the steeds surge forward.

A halcyon wave washes over her, And her single-serving companions, Reducing uninhibited guffaws, Shrieks, and vulgar whispers To hushed murmurs, As hooves and her heart Both pound with earnest impatience.

She presses her cheek to the cold metal tent pole, Trying to steady herself

Against the weight of the world And the trembling of the ground. Time slows, and she notices then How the crisp fall air mingles with the scent Of freshly cut hay, perfume, and gin.

How difficult it is to break away, Like a stallion before the gate opens, From these inebriated, carousing strangers, To whom it seems secrets are currency, And from the insalubrious mixture in her hand That has defined her, marked her, Siphoned her health these past years.

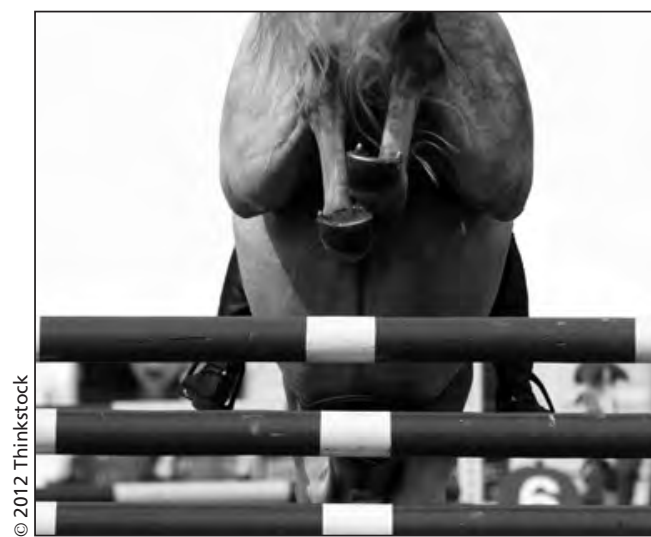

These majestic equines calm her, Their rippling velvet skin concealing The raw power of a million myocytes; Form and function seamlessly married, Whispering of a harmony in nature She now struggles to regain.

\section{Ryan E. Childers MD}

Internal medicine PGY-3

Department of Medicine Johns Hopkins Bayview Medical Center Baltimore, Md.

CMAJ 2012. DOI:10.1503/cmaj.120158 